\title{
Europäischer Tarifbericht des WSI 2007/2008
}

In den Jahren 2007 und 2008 hat die Entwicklung der Löhne in ganz Europa deutlich an Dynamik gewonnen. Dies ist eine Reaktion auf den ökonomischen Aufschwung, der mit steigenden Wachstumsraten und rückläufigen Arbeitslosenzahlen die Verhandlungsposition der Arbeitnehmer verbessert hat. Hinzu kommt 2008 ein starker Anstieg der Verbraucherpreise. Die Angst vor einer Lohn-PreisSpirale ist jedoch unbegründet, da die Reallohnsteigerungen weiterhin durch die Produktivitätszuwächse gedeckt sind. Da für die kommenden Jahre die Wachstumsprognosen für Europa wieder deutlich geringer ausfallen, gewinnt die Lohnpolitik für die Stabilisierung der Konjunktur zunehmend an Bedeutung.

\section{Ökonomische Rahmenbedingungen der Tarifpolitik}

\subsection{ALLGEMEINE WIRTSCHAFTS- ENTWICKLUNG}

Die Tarifauseinandersetzungen des Jahres 2007 fanden innerhalb Europas vor dem Hintergrund einer prosperierenden Wirtschaft mit relativ hohen Wachstumsraten statt. In vielen europäischen Ländern hat sich das Tempo des Aufschwungs im Vergleich zum Vorjahr jedoch bereits wieder etwas verlangsamt. Innerhalb der Europäischen Union (EU 27) lag das durchschnittliche Wachstum des Bruttoinlandsproduktes (BIP) mit 2,8 \% im Jahr 2007 leicht unterhalb des Wachstums im Jahr 2006, in dem noch $3,1 \%$ erreicht wurden (Tabelle 1 ). ${ }^{1}$

Die dynamischste Entwicklung vollzog sich wiederum in den neuen EU-Staaten aus Mittel- und Osteuropa, deren Wachstumsraten in der Regel zwischen $6 \%$ und $7 \%$ lagen und im Falle von Lettland und der Slowakei sogar Spitzenwerte von über $10 \%$ erreichten. Die einzige Ausnahme bildete Ungarn, das mit nur 1,3 \% bereits 2007 einen starken Wachstumseinbruch erlebte. In den alten EU-Staaten waren Irland und Luxemburg mit Wachstumsraten von über $5 \%$ erneut die Spitzenreiter, während Dänemark, Frankreich, Italien und Portugal mit Werten von unter $2 \%$ die schwächste ökonomische Entwicklung aufwiesen. Deutschland befand sich mit einem BIPWachstum von 2,5\% im westeuropäischen Mittelfeld.

Vor dem Hintergrund der internationalen Immobilien- und Finanzmarktkrise und einer sich eintrübenden Weltkonjunktur geht die Europäische Kommission (2008b) in ihrer Frühjahrsprognose von einer deutlichen Abschwächung der konjunkturellen Dynamik aus. Im EU-Durchschnitt wird für 2008 lediglich eine Wachstumsrate von $2 \%$ prognostiziert. In vielen alten EU-Staaten - darunter auch in Deutschland - wird das Wachstum sogar unter $2 \%$ liegen, während Italien sich mit einem BIP-Wachstum von nur 0,5\% bereits am Rande einer Rezession befindet. In den mittel- und osteuropäischen Ländern ist die Wachstumsdynamik ebenfalls stark rückläufig. Allerdings wird eine Reihe von Ländern nach wie vor relativ hohe Wachstumsraten zwischen $5 \%$ und $7 \%$ erreichen.

\subsection{DIE LAGE AUF DEM ARBEITSMARKT}

Die Lage auf dem Arbeitmarkt hat sich im Jahr 2007 angesichts der hohen Wachstumsraten in den meisten EU-Staaten weiter verbessert. Insgesamt betrug die durchschnittliche Arbeitslosenquote in der EU $7,1 \%$ und lag damit einen gesamten Prozentpunkt unterhalb des Vorjahres. Zwischen den einzelnen EU-Staaten haben sich die nationalen Arbeitslosenquoten zwar weiter angenähert, nach wie vor bestehen jedoch signifikante Unterschiede. Die höchste Arbeitslosenquote existierte mit $11,1 \%$ in der Slowakei, gefolgt von Polen mit 9,6\%. Dahinter befand sich eine Gruppe von Ländern mit Arbeitslosenraten um die $8 \%$, zu der neben den südeuropäischen Ländern Griechenland, Spanien und Portugal auch Frankreich und Deutschland gehörten. Die geringste Arbeitslosigkeit herrschte mit einer Quote von $2,9 \%$ in den Niederlanden sowie in Dänemark mit 3,1 \%.

Im Jahr 2008 wird sich die positive Entwicklung auf dem Arbeitsmarkt - wenn auch in einem deutlich geringeren Tempo - in den meisten Ländern weiter fortsetzen. Nach Prognosen der Europäischen Kommission wird die Arbeitslosenquote im EU-Durchschnitt bei 6,8 \% liegen. Im Juni 2008 gab es innerhalb der EU insgesamt 16,3 Mio. offiziell registrierte Arbeitslose. Dies waren 0,6 Mio. weniger als im gleichen Monat des Vorjahres (Eurostat 2008). Eine rückläufige Arbeitslosigkeit wirkt sich in der Regel positiv auf die Verhandlungsmacht der Gewerkschaften aus und lässt damit tendenziell höhere Lohnabschlüsse erwarten. So gelangt der Europäische Gewerkschaftsbund (EGB) in seiner Stellungnahme zur europaweiten Koordinierung der Tarifrunde 2008 zu dem Ergebnis, dass bei einem Rückgang der Arbeitslosigkeit und einem dynamischen Beschäftigungswachstum die Arbeitnehmer weniger erpressbar seien, den Forderungen der Arbeitgeber nachzugeben, dass Lohnzurückhaltung zur Sicherung der Arbeitsplätze vonnöten sei. Auch das Bestreben der Arbeitgeber, bei vollen Auftragsbüchern Arbeitskämpfe zu vermeiden, stärke die Verhandlungsposition der Beschäftigten (EGB 2007).

\footnotetext{
1 Im Folgenden wird, falls nicht anders ausgewiesen, auf Daten aus der AMECO-Datenbank der Europäischen Kommission zurückgegriffen (Europäische Kommission 2008a). Bei den Angaben für 2008 handelt es sich um Prognosen aus dem Frühjahrsgutachten der Europäischen Kommission (Europäische Kommission 2008b). Zur Konjunkturentwicklung in Europa s.a. Projektgruppe Ge meinschaftsdiagnose (2008) und ELNEP (2008).
}

Thorsten Schulten, Dr., Wissenschaftler im WSI in der Hans-Böckler-Stiftung. Arbeitsschwerpunkte: Arbeits- und Tarifpolitik in Europa. e-mail: Thorsten-Schulten@boeckler.de 
In vielen Ländern ist die Zahl der Arbeitslosen allerdings nach wie vor sehr hoch und wirkt sich dort immer noch dämpfend auf die gewerkschaftliche Durchsetzungskraft aus. Hinzu kommt, dass die positive Beschäftigungsdynamik der letzten Jahre in vielen europäischen Ländern mit einer starken Zunahme von prekären Beschäftigungsverhältnissen einhergegangen ist, deren Bestand bei einer stärkeren Konjunktureintrübung wieder gefährdet sein könnte (ELNEP 2008, S. 50ff.).

\subsection{PREISE UND PRODUKTIVITÄT - DER LOHNPOLITISCHE VERTEILUNGSSPIELRAUM}

Während die Konjunkturentwicklung und die Lage auf dem Arbeitsmarkt den allgemeinen ökonomischen Rahmen bilden, orientieren sich die Tarifauseinandersetzungen in Europa vor allem an der Entwicklung der Preise und der Arbeitsproduktivität (Tabelle 2). Die Summe beider Indikatoren bildet den lohnpolitischen Verteilungsspielraum, dessen Ausschöpfung durch entsprechende Lohnerhöhungen den Beschäftigten eine gleichgewichtige Partizipation an der allgemeinen Wirtschaftentwicklung garantiert und das Verhältnis zwischen Arbeits- und Kapitaleinkommen konstant hält. Makroökonomisch gilt die produktivitätsorientierte (Real-)Lohnpolitik als wachstums- und stabilitätsgerecht, da sie die private Konsumnachfrage fördert, ohne die Geldwertstabilität zu gefährden.

Nachdem innerhalb der Europäischen Währungsunion (EWU) der Wechselkurs als Ausgleichsmechanismus für unterschiedliche ökonomische Entwicklungsdynamiken weggefallen ist, wirkt sich die Lohnentwicklung innerhalb der EWU unmittelbar auf die preisliche Wettbewerbsfähigkeit einer nationalen Volkswirtschaft aus. Mittels einer moderaten Lohnpolitik lässt sich deshalb die Wettbewerbsfähigkeit der heimischen Exportindustrie fördern. Dies funktioniert allerdings nur, solange nicht alle Länder die gleiche Strategie verfolgen. In diesem Fall käme es zu einer europäischen Lohnsenkungsspirale mit negativen Folgen für Wachstum und Beschäftigung in der gesamten EWU (Vring 2008). Für eine stabilitätsgerechte Lohnpolitik fordert deshalb z. B. die Europäische Kommission (2005, S. 17), dass „die realen Lohnerhöhungen mit dem mittelfristigen Produktivitätswachstumstrend in Einklang stehen." Auch die europäischen Gewerk-

Tabelle 1: Wachstum und Arbeitslosigkeit in der Europäischen Union 2004-2008 - in \% -

\begin{tabular}{|c|c|c|c|c|c|c|c|c|}
\hline & \multicolumn{4}{|c|}{ Bruttoinlandsprodukt ${ }^{1}$ ) } & \multicolumn{4}{|c|}{ Arbeitslosenquote2) } \\
\hline & 2005 & 2006 & 2007 & 2008 & 2005 & 2006 & 2007 & 2008 \\
\hline \multicolumn{9}{|l|}{ Alte EU-Staaten } \\
\hline Belgien & 1,7 & 2,8 & 2,7 & 1,7 & 8,4 & 8,2 & 7,5 & 7,3 \\
\hline Dänemark & 2,5 & 3,9 & 1,8 & 1,3 & 4,8 & 3,9 & 3,7 & 3,1 \\
\hline Deutschland & 0,8 & 2,9 & 2,5 & 1,8 & 10,7 & 9,8 & 8,4 & 7,3 \\
\hline Finnland & 2,8 & 4,9 & 4,4 & 2,8 & 8,4 & 7,7 & 6,9 & 6,3 \\
\hline Frankreich & 1,7 & 2,0 & 1,9 & 1,6 & 9,2 & 8,3 & 8,0 & 8,1 \\
\hline Griechenland & 3,8 & 4,2 & 4,0 & 3,4 & 9,8 & 8,9 & 8,3 & 8,3 \\
\hline Großbritannien & 1,8 & 2,9 & 3,0 & 1,7 & 4,8 & 5,3 & 5,2 & 5,4 \\
\hline Irland & 6,0 & 5,7 & 5,3 & 2,3 & 4,3 & 4,4 & 4,5 & 5,6 \\
\hline Italien & 0,6 & 1,8 & 1,5 & 0,5 & 7,7 & 6,8 & 6,1 & 6,0 \\
\hline Luxemburg & 5,0 & 6,1 & 5,1 & 3,6 & 4,5 & 4,7 & 4,7 & 4,5 \\
\hline Niederlande & 1,5 & 3,0 & 3,5 & 2,6 & 4,7 & 3,9 & 3,2 & 2,9 \\
\hline Österreich & 2,0 & 3,3 & 3,4 & 2,2 & 5,2 & 4,7 & 4,4 & 4,2 \\
\hline Portugal & 0,9 & 1,3 & 1,9 & 1,7 & 7,6 & 7,7 & 8,0 & 7,9 \\
\hline Schweden & 3,3 & 4,1 & 2,6 & 2,2 & 7,4 & 7,0 & 6,1 & 6,2 \\
\hline Spanien & 3,6 & 3,9 & 3,8 & 2,2 & 9,2 & 8,5 & 8,3 & 9,3 \\
\hline EU 15 & 1,0 & 2,8 & 2,6 & 1,7 & 8,1 & 7,7 & 7,0 & 6,8 \\
\hline \multicolumn{9}{|l|}{ Neue EU-Staaten } \\
\hline Bulgarien & 6,2 & 6,3 & 6,2 & 5,8 & 10,1 & 9,0 & 6,9 & 6,0 \\
\hline Estland & 10,2 & 11,2 & 7,1 & 2,7 & 7,9 & 5,9 & 4,7 & 6,0 \\
\hline Lettland & 10,6 & 12,2 & 10,3 & 3,8 & 8,9 & 6,8 & 6,0 & 6,4 \\
\hline Litauen & 7,9 & 7,7 & 8,8 & 6,1 & 8,3 & 5,6 & 4,3 & 4,5 \\
\hline Malta & 3,4 & 3,4 & 3,8 & 2,6 & 7,3 & 7,3 & 6,4 & 6,3 \\
\hline Polen & 3,6 & 6,2 & 6,5 & 5,3 & 17,7 & 13,8 & 9,6 & 7,1 \\
\hline Rumänien & 4,2 & 7,9 & 6,0 & 6,2 & 7,2 & 7,3 & 6,4 & 6,1 \\
\hline Slowakei & 6,6 & 8,5 & 10,4 & 7,0 & 16,3 & 13,4 & 11,1 & 9,8 \\
\hline Slowenien & 4,1 & 5,7 & 6,1 & 4,2 & 6,5 & 6,0 & 4,8 & 4,7 \\
\hline Tschechien & 6,4 & 6,4 & 6,5 & 4,7 & 7,9 & 7,1 & 5,3 & 4,5 \\
\hline Ungarn & 4,1 & 3,9 & 1,3 & 1,9 & 7,2 & 7,5 & 7,4 & 8,3 \\
\hline Zypern & 3,9 & 4,0 & 4,4 & 3,7 & 5,2 & 4,6 & 3,9 & 3,7 \\
\hline Gesamte EU 27 & 1,9 & 3,1 & 2,8 & 2,0 & 8,9 & 8,1 & 7,1 & 6,8 \\
\hline \multicolumn{9}{|c|}{$\begin{array}{l}\text { 1) Bruttoinlandsprodukt }=\text { Veränderung des } \mathrm{BIP} \text { in \% gegenüber dem Vorjahr. } \\
\text { 2) Arbeitslosenquote = Anzahl der Arbeitslosen in \% der zivilen Erwerbsbevölkerung (Eurostat-Definition). } \\
\text { Angaben für } 2008=\text { Frühjahrsprognose } 2008 \text { der Europäischen Kommission. } \\
\text { Quelle: Europäische Kommission (2008a). }\end{array}$} \\
\hline
\end{tabular}

schaften haben sich bereits seit Ende der 1990er Jahre als Zielmarke für eine europäische Koordinierung der Tarifpolitik auf eine produktivitätsorientierte (Real-) Lohnpolitik verständigt, die die nationalen Verteilungsspielräume ausschöpft, um auf diese Weise gegenseitiges Lohndumping zu verhindern (EGB 2007).

Bei der Definition des tarifpolitischen Verteilungsspielraumes als Summe aus Preis- und Produktivitätsentwicklung ist in der Wirtschaftswissenschaft strittig, welche konkreten Indikatoren insbesondere für die Preisentwicklung verwendet werden sollen. Gegenüber möglichen alternativen Indikatoren (wie z. B. der Kerninflationsrate, der EZB-Zielinflationsrate oder der Entwicklung der Erzeugerpreise) wird im Folgenden bei der Bestimmung der nationalen Verteilungsspielräume die jeweils nationale Entwicklung der Verbraucherpreise zugrunde gelegt. Aus Arbeitnehmersicht stellen diese den wichtigsten ökonomi- schen Indikator dar und bilden damit für die Gewerkschaften die bedeutsamste Orientierungsgröße bei den Tarifauseinandersetzungen.

Im Jahr 2007 hat sich die Entwicklung der (Verbraucher-)Preise innerhalb der EU kaum verändert. Der harmonisierte Verbraucherpreisindex (HVPI) stieg im EUDurchschnitt mit 2,4 \% nur unwesentlich schneller als in den Vorjahren (Tabelle 2). In den alten EU-Staaten bewegte sich die Preisentwicklung zwischen $1,6 \%$ in Finnland und den Niederlanden und 3,0\% in Griechenland. Nach wie vor deutlich höhere Inflationsraten hatten einige mittelund osteuropäische Länder, wobei Lettland mit 10,1 \% den höchsten Wert aufwies. Für 2008 erwartet die Europäische Kommission erstmals seit Langem wieder einen kräftigen Anstieg der Preise auf EU-weit insgesamt 3,6\%. Verantwortlich hierfür ist in erster Linie der starke Anstieg der internationalen Energie- und Nahrungsmittel- 


\begin{tabular}{|c|c|c|c|c|c|c|c|c|c|c|c|c|}
\hline & \multicolumn{4}{|c|}{ Preise ${ }^{1)}$} & \multicolumn{4}{|c|}{ Arbeitsproduktivität2) } & \multicolumn{4}{|c|}{$\begin{array}{c}\text { Lohnpolitischer } \\
\text { Verteilungsspielraum } 3 \text { ) }\end{array}$} \\
\hline & 2005 & 2006 & 2007 & 2008 & 2005 & 2006 & 2007 & 2008 & 2005 & 2006 & 2007 & 2008 \\
\hline \multicolumn{13}{|l|}{ Alte EU-Staaten } \\
\hline Belgien & 2,5 & 2,3 & 1,8 & 3,6 & 0,4 & 1,6 & 1,0 & 0,8 & 2,9 & 3,9 & 2,8 & 4,4 \\
\hline Dänemark & 1,7 & 1,9 & 1,7 & 3,3 & 1,6 & 2,2 & 0,0 & 1,1 & 3,3 & 4,1 & 1,7 & 4,4 \\
\hline Deutschland & 1,9 & 1,8 & 2,3 & 2,9 & 1,3 & 2,7 & 1,0 & 0,8 & 3,2 & 4,5 & 3,3 & 3,7 \\
\hline Finnland & 0,8 & 1,3 & 1,6 & 3,4 & 1,4 & 3,0 & 2,1 & 1,4 & 2,2 & 4,3 & 3,7 & 4,8 \\
\hline Frankreich & 1,9 & 1,9 & 1,6 & 3,0 & 1,4 & 1,2 & 0,6 & 0,9 & 3,3 & 3,1 & 2,2 & 3,9 \\
\hline Griechenland & 3,5 & 3,3 & 3,0 & 3,7 & 2,3 & 1,7 & 2,7 & 2,2 & 5,8 & 5,0 & 5,7 & 5,9 \\
\hline Großbritannien & 2,1 & 2,3 & 2,3 & 2,8 & 0,8 & 2,0 & 2,3 & 1,7 & 2,9 & 4,3 & 4,6 & 4,5 \\
\hline Irland & 2,2 & 2,7 & 2,9 & 3,3 & 1,3 & 1,4 & 1,6 & 1,6 & 3,5 & 4,1 & 4,5 & 4,9 \\
\hline Italien & 2,2 & 2,2 & 2,0 & 3,0 & 0,4 & 0,1 & 0,5 & 0,1 & 2,6 & 2,3 & 2,5 & 3,1 \\
\hline Luxemburg & 3,8 & 3,0 & 2,7 & 4,2 & 2,1 & 2,3 & 0,6 & $-0,4$ & 5,9 & 5,3 & 3,3 & 3,8 \\
\hline Niederlande & 1,5 & 1,7 & 1,6 & 2,7 & 1,8 & 1,2 & 1,1 & 1,3 & 3,3 & 2,9 & 2,7 & 4,0 \\
\hline Österreich & 2,1 & 1,7 & 2,2 & 3,0 & 1,2 & 1,7 & 1,4 & 1,3 & 3,3 & 3,4 & 3,6 & 4,3 \\
\hline Portugal & 2,1 & 3,0 & 2,4 & 2,8 & 0,9 & 0,6 & 1,7 & 1,0 & 3,0 & 3,6 & 4,1 & 3,8 \\
\hline Schweden & 0,8 & 1,5 & 1,7 & 2,4 & 3,0 & 2,3 & 0,3 & 1,5 & 3,8 & 3,8 & 2,0 & 3,9 \\
\hline Spanien & 3,4 & 3,6 & 2,8 & 3,8 & 0,4 & 0,7 & 0,8 & 0,9 & 3,8 & 4,3 & 3,6 & 4,7 \\
\hline EU 15 & 2,1 & 2,2 & 2,2 & 3,1 & 1,1 & 1,6 & 1,1 & 1,0 & 3,2 & 3,8 & 3,3 & 4,1 \\
\hline \multicolumn{13}{|l|}{ Neue EU-Staaten } \\
\hline Bulgarien & 6,0 & 7,4 & 7,6 & 9,9 & 3,5 & 2,9 & 3,3 & 4,1 & 9,5 & 10,3 & 10,9 & 14,0 \\
\hline Estland & 4,1 & 4,4 & 6,7 & 9,5 & 8,3 & 5,3 & 6,6 & 3,7 & 12,4 & 9,7 & 13,3 & 13,2 \\
\hline Lettland & 6,9 & 6,6 & 10,1 & 15,8 & 8,7 & 7,2 & 6,6 & 4,3 & 15,6 & 13,8 & 16,7 & 20,1 \\
\hline Litauen & 2,7 & 3,8 & 5,8 & 10,1 & 5,3 & 5,9 & 6,7 & 6,0 & 8,0 & 9,7 & 12,5 & 16,1 \\
\hline Malta & 2,5 & 2,6 & 0,7 & 3,4 & 2,0 & 2,2 & 1,1 & 1,3 & 4,5 & 4,8 & 1,8 & 4,7 \\
\hline Polen & 2,2 & 1,3 & 2,6 & 4,3 & 1,3 & 2,9 & 1,9 & 2,6 & 3,5 & 4,2 & 4,5 & 6,9 \\
\hline Rumänien & 9,1 & 6,6 & 4,9 & 7,6 & 5,8 & 4,9 & 4,7 & 5,2 & 14,9 & 11,5 & 9,6 & 12,8 \\
\hline Slowakei & 2,8 & 4,3 & 1,9 & 3,8 & 5,1 & 6,1 & 8,1 & 5,5 & 7,9 & 10,4 & 10,0 & 9,3 \\
\hline Slowenien & 2,5 & 2,5 & 3,8 & 5,4 & 4,0 & 4,5 & 3,3 & 3,3 & 6,5 & 7,0 & 7,1 & 8,7 \\
\hline Tschechien & 1,6 & 2,1 & 3,0 & 6,2 & 5,2 & 4,5 & 4,6 & 3,5 & 6,8 & 6,6 & 7,6 & 9,7 \\
\hline Ungarn & 3,5 & 4,0 & 7,9 & 6,3 & 3,7 & 2,9 & 1,5 & 3,1 & 7,2 & 6,9 & 9,4 & 9,4 \\
\hline Zypern & 2,0 & 2,2 & 2,2 & 3,8 & 0,3 & 2,3 & 1,1 & 2,2 & 2,3 & 4,5 & 3,3 & 6,0 \\
\hline Gesamte EU 27 & 2,3 & 2,3 & 2,4 & 3,6 & 1,2 & 1,7 & 1,3 & 1,2 & 3,5 & 4,0 & 3,7 & 4,8 \\
\hline \multicolumn{13}{|c|}{$\begin{array}{l}\text { 1) Preise = Harmonisierter Verbraucherpreisindex (HVPI), Veränderung in \% gegenüber dem Vorjahr. } \\
\text { 2) Arbeitsproduktivität = Reales BIP pro Kopf, Veränderung in \% gegenüber dem Vorjahr. } \\
\text { 3) Tarifpolitischer Verteilungsspielraum = Summe aus Preis- und Arbeitsproduktivitätsentwicklung. } \\
\text { Angaben für } 2008=\text { Frühjahrsprognose } 2008 \text { der Europäischen Kommission. }\end{array}$} \\
\hline \multicolumn{13}{|c|}{ Quelle: Europäische Kommission (2008a); Berechnungen des WSI. } \\
\hline
\end{tabular}

preise (Projektgruppe Gemeinschaftsdiagnose 2008, S.22)

Das Wachstum der Arbeitsproduktivität hat im Jahr 2007 im EU-Durchschnitt mit $1,3 \%$ im Vergleich zu 1,7 \% im Vorjahr wieder an Tempo verloren (Tabelle 2). Mit deutlich höheren Wachstumsraten von $3 \%$ bis zu $8 \%$ bekräftigten die meisten mittel- und osteuropäischen EU-Staaten ihren anhaltenden ökonomischen Aufholprozess. In den alten EU-Staaten variierten die Produktivitätszuwächse zwischen $2,7 \%$ in Griechenland und 0,3 \% in Schweden. In Dänemark kam es sogar zu einer Stagnation bei der Produktivitätsentwicklung. Für 2008 erwartet die Europäische Kommission eine Entwicklung, die sich im EU-Durchschnitt auf ähnlichem Niveau wie 2007 bewegt.

Aus der Gesamtsumme von Preis- und Produktivitätsentwicklung ergib sich im Durchschnitt für die alten EU-Staaten ein lohnpolitischer Verteilungsspielraum von

\section{Lohnentwicklung und Ausschöpfung nationaler Verteilungsspielräume}

Bis heute existiert innerhalb Europas keine verlässliche Statistik über die Entwicklung der Tariflöhne in Europa. ${ }^{2}$ Der einzige bis-

lang zur Verfügung stehende offizielle Indikator ist der Tariflohnindex für die EWU, der von der Europäischen Zentralbank auf der Grundlage nicht harmonisierter nationaler Statistiken berechnet wird. ${ }^{3}$ Die Tariflohnentwicklung in der EWU ist mit durchschnittlichen Steigerungsraten zwischen 2,1 \% und 2,3 \% seit Ende der 1990er Jahre erstaunlich konstant verlaufen $(A b$ bildung 1). Im Jahr 2007 stiegen Tariflöhne in der Eurozone um 2,2 \%. Demgegenüber weisen die Tariferhöhungen in Deutschland eine erheblich höhere Schwankungsbreite aus. Während 2007 die deutschen Tariflöhne mit 2,2\% im gleichen Tempo wie in der gesamten EWU zunahmen, lagen die Tarifabschlüsse in den Jahren zuvor deutlich unterhalb des EWU-Durchschnittes.

Hinzu kommt, dass in der Eurozone insgesamt eine relativ starke positive Lohndrift vorherrscht, d.h. dass die Effektivlöhne in der Regel deutlich stärker steigen als die Tariflöhne ansteigen (Schulten 2007, S. 478). In Deutschland war die Lohndrift in den letzten Jahren hingegen zumeist negativ, sodass die Beschäftigten im Durchschnitt geringere Lohnsteigerungen bekamen, als in den Tarifverträgen vereinbart wurden. Letzteres ist das Ergebnis einer rückläufigen Tarifbindung sowie der zunehmenden Möglichkeiten, auf betrieblicher Ebene von tarifvertraglichen Stan-

\footnotetext{
2 Seit einigen Jahren veröffentlicht das European Industrial Relations Observatory (EIRO) regelmäßig Daten über die Entwicklung der Tariflöhne in Europa (vgl. zuletzt Carley 2008a). Eine Überprüfung der vom EIRO verwendeten nationalen Ursprungsdaten macht jedoch deutlich, dass diese große methodische Mängel aufweisen und nicht miteinander vergleichbar sind. Zum einen werden in den EIRO-Daten mitunter sehr unterschiedliche Bereiche und Zeiträume erfasst. Zum anderen werden aufgrund fehlender nationaler Tariflohnstatistiken oft Datenquellen verwendet, die faktisch keine Tarif-, sondern Effektivlohnentwicklungen darstellen. Qualitative Berichte über die Tarifauseinandersetzungen in den einzelnen EU-Staaten finden sich in den laufenden nationalen Beiträgen zum EIRO (http://www.eurofound. europa.eu/eiro) sowie in dem gemeinsam vom Europäischen Gewerkschaftsinstitut und dem Amsterdam Institute for Advanced Labour Studies (AIAS) herausgegebenen Collective Bargaining Newsletter (http://www.etui-rehs.org/research/ Publications/Newsletters/Collective-bargainingnewsletter).

3 Grundlage hierfür sind nationale Daten aus neun EWU-Staaten, die insgesamt $97 \%$ des EuroRaums abdecken. Die EZB veröffentlicht allerdings nur die aggregierten Tarifdaten für die gesamte Eurozone.
} 
dards nach unten abzuweichen (Bispinck 2007).

Die im Folgenden verwendeten Daten beziehen sich ausschließlich auf die Entwicklung der Effektivlöhne. ${ }^{4}$ Angesichts der für die meisten EU-Staaten zu unterstellenden positiven Lohndrift kann daher von diesen nur bedingt auf die Bedeutung der Tarifpolitik oder den Einfluss der Gewerkschaften geschlossen werden.

\subsection{ENTWICKLUNG DER NOMINAL- UND REALLÖHNE}

Im EU-Durchschnitt stiegen die Nominallöhne 2007 um 2,9\% und lagen damit leicht über dem Vorjahreszuwachs von 2,7 \% (Tabelle 3). Für 2008 erwartet die Europäische Kommission eine deutlich höhere Nominallohnsteigerung von durchschnittlich 3,8\%. Der starke Anstieg kann zum einen damit erklärt werden, dass die Löhne sich immer in einem gewissen time lag zur allgemeinen Konjunktur herausbilden, sodass die Lohnentwicklung 2008 immer noch von den starken Aufschwungjahren 2006 und 2007 beeinflusst wird. Zum anderen ist die Lohnentwicklung im Jahr 2008 auch eine Reaktion auf die deutlich gestiegenen Preissteigerungsraten in Europa.

Entsprechend ihrer besonders dynamischen ökonomischen Entwicklung wurden die höchsten Nominallohnzuwächse in den mittel- und osteuropäischen Staaten erzielt. Der Spitzenreiter des Jahres 2007 war Lettland mit einer Nominallohnsteigerung von 33,2 \%, gefolgt von Estland mit 26,5\% und Rumänien mit 20,2\%. In den übrigen Ländern bewegten sich die Zuwächse zwischen $6 \%$ und $18 \%$. Aus der Gruppe der alten EU-Staaten konnte Griechenland mit $7,2 \%$ den höchsten Nominallohnanstieg verzeichnen, gefolgt von Irland mit 5,8 \%. Darüber hinaus lagen die Steigerungsraten in den meisten alten EU-Staaten zwischen $2,5 \%$ und $4 \%$. Lediglich Deutschland fällt mit einer Nominallohnsteigerung von nur 1,1\% einmal mehr aus dem Rahmen und war auch 2007 wieder mit Abstand das lohnpolitische Schlusslicht in Europa.

4 Streng genommen werden in der AMECO-Datenbank der Europäischen Kommission keine „Löhne", sondern "Arbeitnehmerentgelte" (definiert als Bruttolöhne plus Arbeitgeberanteile zur Sozialversicherung) ausgewiesen. Der hier im Folgenden verwendete weite Lohnbegriff umfasst demnach die gesamten Arbeitskosten.

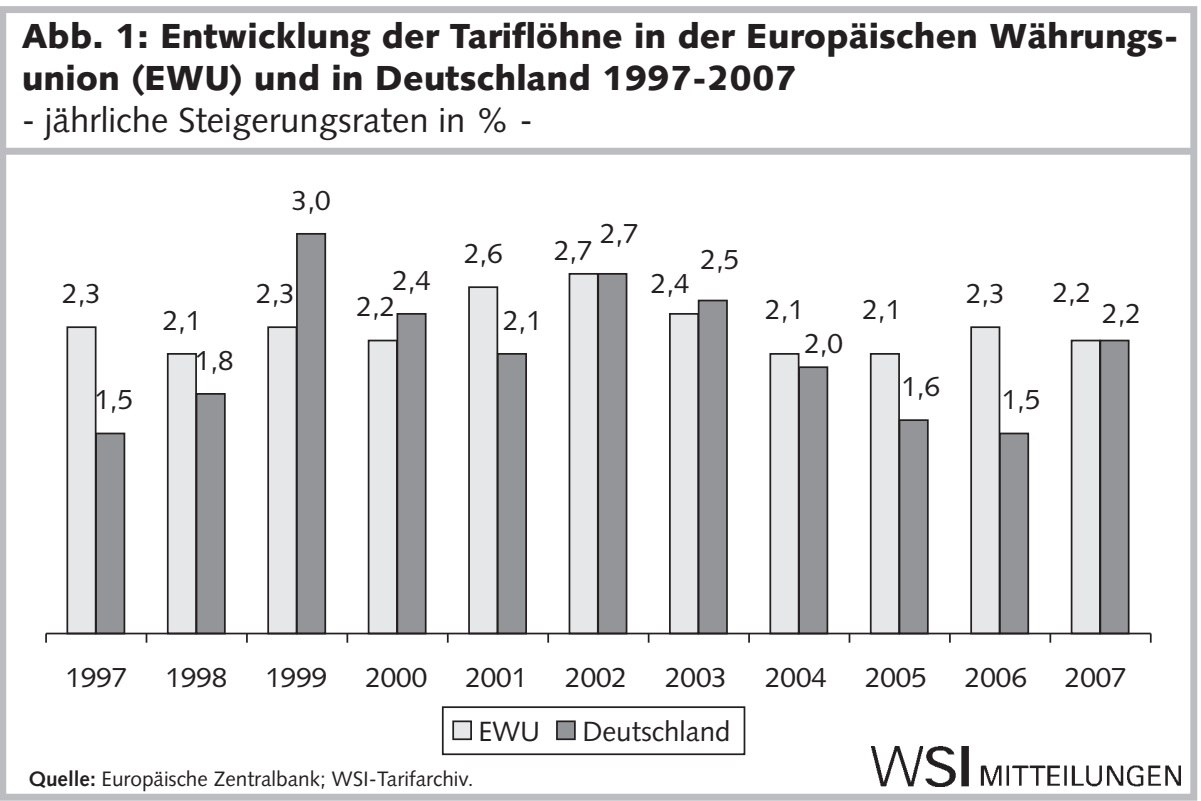

Tabelle 3: Lohnentwicklung und Verteilungsbilanz in der Europäischen Union 2005-2008 - in \% -

\begin{tabular}{|c|c|c|c|c|c|c|c|c|c|c|c|c|}
\hline & \multicolumn{4}{|c|}{ Nominallöhne ${ }^{1)}$} & \multicolumn{4}{|c|}{ Reallöhne2) } & \multicolumn{4}{|c|}{ Verteilungsbilanz ${ }^{3)}$} \\
\hline & 2005 & 2006 & 2007 & 2008 & 2005 & 2006 & 2007 & 2008 & 2005 & 2006 & 2007 & 2008 \\
\hline \multicolumn{13}{|l|}{ Alte EU-Staaten } \\
\hline Belgien & 1,9 & 3,2 & 3,0 & 3,5 & $-0,6$ & 0,9 & 1,2 & $-0,1$ & $-1,0$ & $-0,7$ & 0,2 & $-0,9$ \\
\hline Dänemark & 3,5 & 3,9 & 3,7 & 4,6 & 1,8 & 2,1 & 2,0 & 1,3 & 0,2 & $-0,1$ & 2,0 & 0,2 \\
\hline Deutschland & 0,5 & 1,6 & 1,1 & 2,1 & $-1,4$ & $-0,2$ & $-1,1$ & $-0,8$ & $-2,7$ & $-2,9$ & $-2,1$ & $-1,6$ \\
\hline Finnland & 3,8 & 2,9 & 3,3 & 5,5 & 3,0 & 1,6 & 1,7 & 2,1 & 1,6 & $-1,4$ & $-0,4$ & 0,7 \\
\hline Frankreich & 3,2 & 3,1 & 3,0 & 3,0 & 1,3 & 1,2 & 1,3 & 0,0 & $-0,1$ & 0,0 & 0,7 & $-0,9$ \\
\hline Griechenland & 6,1 & 6,3 & 7,2 & 7,4 & 2,6 & 3,0 & 4,2 & 3,7 & 0,3 & 1,3 & 1,5 & 1,5 \\
\hline Großbritannien & 4,3 & 5,0 & 3,9 & 4,3 & 2,3 & 2,7 & 1,5 & 1,5 & 1,5 & 0,7 & $-0,8$ & $-0,2$ \\
\hline Irland & 5,0 & 4,5 & 5,8 & 4,9 & 2,9 & 1,8 & 3,0 & 1,6 & 1,6 & 0,4 & 1,4 & 0,0 \\
\hline Italien & 3,2 & 2,5 & 1,9 & 3,8 & 1,0 & 0,3 & $-0,1$ & 0,8 & 0,6 & 0,2 & $-0,6$ & 0,7 \\
\hline Luxemburg & 3,8 & 4,5 & 4,0 & 3,2 & 0,1 & 1,6 & 1,3 & $-1,0$ & $-2,0$ & $-0,7$ & 0,7 & $-0,6$ \\
\hline Niederlande & 1,6 & 2,4 & 2,7 & 3,6 & 0,1 & 0,7 & 1,1 & 0,9 & $-1,7$ & $-0,5$ & 0,0 & $-0,4$ \\
\hline Österreich & 2,6 & 2,4 & 2,6 & 3,2 & 0,4 & 0,8 & 0,4 & 0,2 & $-0,8$ & $-0,9$ & $-1,0$ & $-1,1$ \\
\hline Portugal & 2,9 & 2,4 & 2,1 & 2,6 & 0,8 & $-0,7$ & $-0,3$ & $-0,2$ & $-0,1$ & $-1,3$ & $-2,0$ & $-1,2$ \\
\hline Schweden & 3,1 & 2,2 & 4,2 & 4,6 & 2,3 & 0,7 & 2,5 & 2,1 & $-0,7$ & $-1,6$ & 2,2 & 0,6 \\
\hline Spanien & 2,9 & 3,0 & 3,6 & 4,3 & $-0,5$ & $-0,6$ & 0,7 & 0,5 & $-0,9$ & $-1,3$ & $-0,1$ & $-0,4$ \\
\hline EU 15 & 2,6 & 2,9 & 2,8 & 3,5 & 0,5 & 0,7 & 0,6 & 0,4 & $-0,6$ & $-0,9$ & $-0,5$ & $-0,6$ \\
\hline \multicolumn{13}{|l|}{ Neue EU-Staaten } \\
\hline Bulgarien & 5,9 & 7,4 & 17,9 & 13,7 & $-0,1$ & 0,0 & 10,4 & 3,8 & $-3,6$ & $-2,9$ & 7,1 & $-0,3$ \\
\hline Estland & 11,0 & 14,0 & 26,5 & 13,6 & 6,9 & 9,6 & 19,7 & 4,0 & $-1,4$ & 4,3 & 13,1 & 0,3 \\
\hline Lettland & 25,3 & 23,6 & 33,2 & 21,0 & 18,4 & 17,0 & 23,1 & 5,2 & 9,7 & 9,8 & 16,5 & 0,9 \\
\hline Litauen & 11,5 & 15,1 & 14,1 & 15,0 & 8,9 & 11,3 & 8,3 & 4,9 & 3,6 & 5,4 & 1,6 & $-1,1$ \\
\hline Malta & 2,0 & 3,3 & 1,5 & 3,0 & $-0,6$ & 0,7 & 0,8 & $-0,4$ & $-2,6$ & $-1,5$ & $-0,3$ & $-1,7$ \\
\hline Polen & 1,6 & 1,9 & 8,1 & 8,0 & $-0,6$ & 0,6 & 5,5 & 3,6 & $-1,9$ & $-2,3$ & 3,6 & 1,0 \\
\hline Rumänien & 22,1 & 17,8 & 20,2 & 18,1 & 13,0 & 11,2 & 15,3 & 10,5 & 7,2 & 6,3 & 10,6 & 5,3 \\
\hline Slowakei & 9,7 & 7,9 & 8,3 & 8,4 & 6,9 & 3,7 & 6,4 & 4,6 & 1,8 & $-2,4$ & $-1,7$ & $-0,9$ \\
\hline Slowenien & 5,3 & 5,5 & 6,2 & 7,8 & 2,9 & 2,9 & 2,5 & 2,3 & $-1,1$ & $-1,6$ & $-0,8$ & $-1,0$ \\
\hline Tschechien & 4,6 & 6,2 & 7,0 & 7,2 & 3,0 & 4,1 & 4,0 & 0,9 & $-2,2$ & $-0,4$ & $-0,6$ & $-2,6$ \\
\hline Ungarn & 7,1 & 8,4 & 8,4 & 6,9 & 3,6 & 4,4 & 0,4 & 0,7 & $-0,1$ & 1,5 & $-1,1$ & $-2,4$ \\
\hline Zypern & 1,8 & 2,7 & 3,5 & 3,5 & $-0,3$ & 0,5 & 1,3 & $-0,3$ & $-0,6$ & $-1,8$ & 0,2 & $-2,5$ \\
\hline Gesamte EU 27 & 2,7 & 2,7 & 2,9 & 3,8 & 0,4 & 0,4 & 0,5 & 0,2 & $-0,8$ & $-1,3$ & $-0,8$ & $-1,0$ \\
\hline \multicolumn{13}{|c|}{$\begin{array}{l}\text { 1) Nominallöhne = Nominaleinkommen aus unselbstständiger Arbeit (einschl. Arbeitgeberbeiträge zur Sozialversicherung) } \\
\text { pro Kopf, Veränderung in \% gegenüber dem Vorjahr. } \\
\text { 2) Reallöhne = Nominaleinkommen aus unselbstständiger Arbeit (einschl. Arbeitgeberbeiträge zur Sozialversicherung) pro Kopf } \\
\text { abzüglich Preisentwicklung (Harmonisierter Verbraucherpreisindex), Veränderung in \% gegenüber dem Vorjahr. } \\
\text { 3) Verteilungsbilanz = Saldo des jährlichen Nominalzuwachs und des lohnpolitischen Verteilungsspielraums (vgl. Tabelle 2) } \\
\text { in Prozentpunkten. } \\
\text { Angaben für } 2008 \text { = Frühjahrsprognose } 2008 \text { der Europäischen Kommission. } \\
\text { Quelle: Europäische Kommission (2008a); Berechnungen des WSI. }\end{array}$} \\
\hline
\end{tabular}




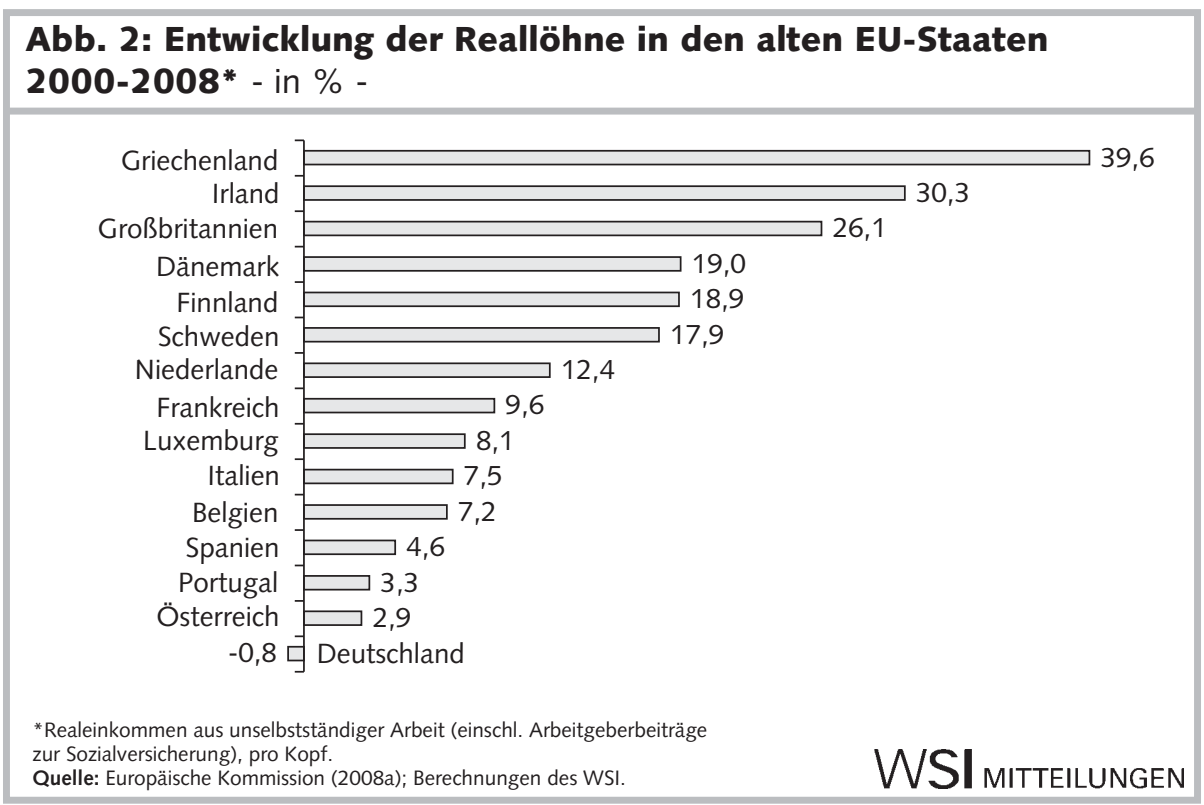

Obwohl die Nominallöhne 2008 mit 2,1\% deutlich stärker ansteigen, wird nach den Prognosen der Europäischen Kommission Deutschland auch in diesem Jahr seine lohnpolitische Sonderstellung in Europa nicht verlieren und sich wiederum am Ende der europäischen Lohnskala bewegen. Dies ist umso erstaunlicher, da die Tariflöhne in Deutschland im ersten Halbjahr 2008 um 3,3 \% angestiegen sind und damit den höchsten Wert seit mehr als zehn Jahren erreicht haben (Bispinck/WSI-Tarifarchiv 2008, S. 457). Während die Lohndrift in konjunkturellen Boomphasen in der Regel einen positiven Verlauf nimmt, bleibt sie in Deutschland erstmalig trotz Konjunkturaufschwung negativ.
Bei den um die Verbraucherpreise bereinigten Reallöhnen kam es im Jahr 2007 im EU-Durchschnitt zu einem leichten Anstieg um $0,5 \%$, der sich ungefähr auf dem Niveau der Vorjahre bewegt (Tabelle 3). In den mittel- und osteuropäischen Ländern konnten dabei auch bei den Reallöhnen teilweise zweistellige Zuwachsraten erzielt werden, wobei erneut Lettland mit 23,1\% die Spitzenposition einnimmt. Auch für die alten EU-Staaten gilt, dass diejenigen Länder mit den höchsten Nominallohnzuwächsen in der Tendenz auch die größten Reallohnsteigerungen aufweisen. So wuchsen die Reallöhne in Irland um 3 \% und in Griechenland sogar um 4,2\%. In den meisten übrigen westeuropäsichen Ländern

\section{Abb. 3: Entwicklung der Reallöhne in den neuen EU-Staaten 2000-2008* - in \% -}

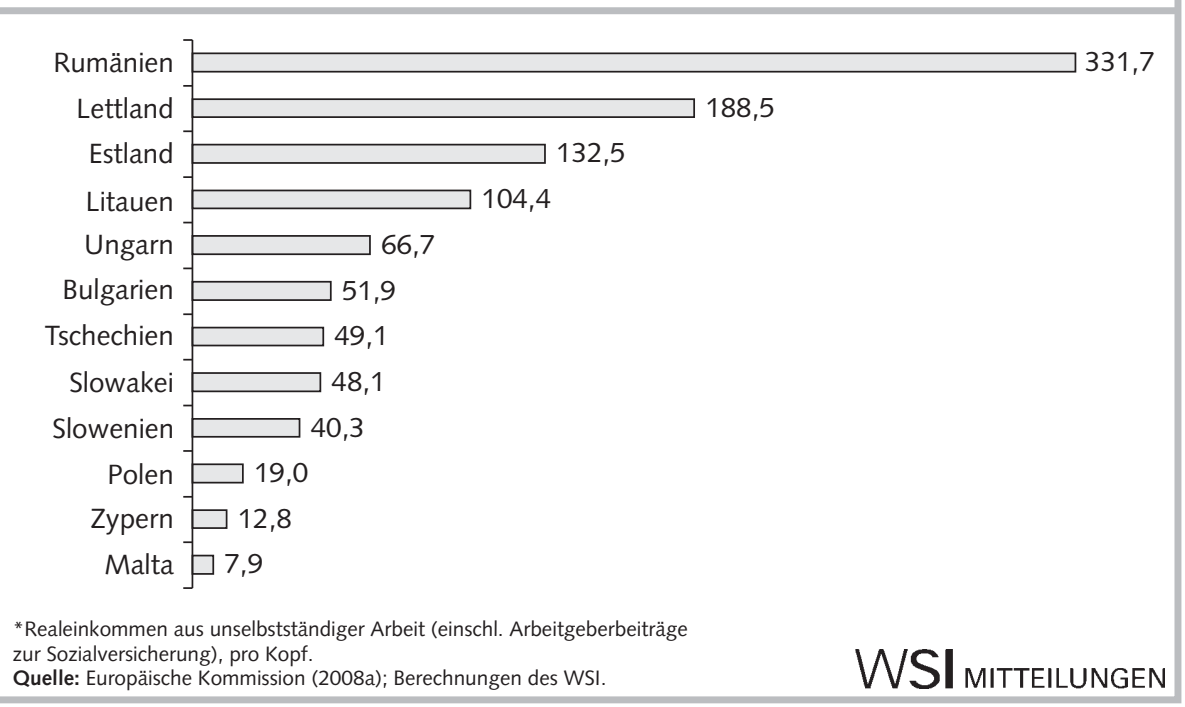

variierten die Zuwächse hingegen zwischen $1 \%$ und $2 \%$. Für 2008 erwartet die Europäische Kommission, dass die Entwicklung der Löhne in fast allen EU-Staaten die hohen Preissteigerungsraten kompensieren kann und - wenn auch in bescheidenerem Maße als im Vorjahr - Reallohnzuwächse sicherstellt.

Im Gegensatz hierzu wird die lohnpolitische Sonderstellung Deutschlands im Hinblick auf die Reallöhne noch einmal besonders prononciert. Als einziges Land in Europa verzeichnete Deutschland 2007 mit einem Minus von 1,1\% einen deutlichen Reallohnverlust und drückte damit die gesamte Entwicklung innerhalb der EU nach unten. Noch nie zuvor in der Geschichte der Bundesrepublik ist ein konjunktureller Aufschwung mit einem anhaltenden Rückgang der Reallöhne einhergegangen (Logeay/Zwiener 2008). Die Europäische Kommission erwartet, dass sich angesichts einer prognostizierten Reallohnentwicklung von minus $0,8 \%$ auch im Jahr 2008 an dieser Situation nichts ändern wird.

Die lohnpolitische Sonderstellung Deutschlands zeigt sich schließlich auch in einer mittelfristigen Perspektive. Vergleicht man die Reallohnentwicklung in Europa seit dem Jahr 2000, so ist Deutschland wiederum das einzige Land, das mit minus $0,8 \%$ Reallohnverluste zu verzeichnen hat, während im gleichen Zeitraum die Reallöhne in Frankreich um 9,6 \%, in Schweden um 17,9\% und in Großbritannien sogar um 26,1\% gestiegen sind (Abbildung 2). Auf der Grundlage eines äußerst niedrigen Ausgangsniveaus symbolisieren die extrem hohen Reallohnzuwächse in den mittelund osteuropäischen Staaten, die seit 2000 im Baltikum mehr als 100 \%, in Rumänien sogar mehr als $300 \%$ ausmachten, den anhaltenden ökonomischen Aufholprozess gegenüber Westeuropa (Abbildung 3).

\subsection{AUSSCHÖPFUNG DER VERTEILUNGSSPIELRÄUME}

Zur Beurteilung der nationalen Lohnentwicklung muss diese ins Verhältnis zu der jeweiligen nationalen ökonomischen Entwicklung gesetzt werden. Ein wesentlicher Indikator hierfür ist die Frage, inwieweit es der Lohnpolitik gelingt, den Verteilungsspielraum aus Preis- und Produktivitätsentwicklung auszuschöpfen. Der Ausschöpfungsgrad kann hierbei durch die Verteilungsbilanz, d.h. dem Saldo aus Nomi- 
nallohnentwicklung und lohnpolitischem Verteilungsspielraum, gemessen werden (Tabelle 3).

Wie bereits in den vorherigen Jahren, war die Verteilungsbilanz auch 2007 in Europa insgesamt negativ. Im EU-Durchschnitt blieb die Lohnentwicklung 0,8 Prozentpunkte hinter dem Verteilungsspielraum zurück. Zwölf von $27 \mathrm{EU}$ Staaten wiesen eine negative Verteilungsbilanz auf, wobei in Deutschland, Portugal, der Slowakei und Ungarn die Löhne am deutlichsten hinter dem Verteilungsspielraum zurückblieben. Eine extrem expansive Lohnentwicklung vollzog sich in den osteuropäischen Staaten Estland, Lettland und Rumänien, wo die nationalen Verteilungsspielräume um mehr als zehn Prozentpunkte übertroffen wurden und es damit zu einer aktiven Umverteilung zugunsten der Lohneinkommen kam. In den alten EU-Staaten war lediglich in Dänemark und in Schweden mit einer positiven Verteilungsbilanz von 2,0 bzw. 2,2 Prozentpunkten die Lohnentwicklung leicht expansiv.

Im Jahr 2008 wird sich trotz höherer Nominallohnsteigerungen der negative Verteilungstrend weiter fortsetzen, wobei die Löhne im EU-Durchschnitt um einen Prozentpunkt hinter den Verteilungsspielräumen zurückbleiben. Mit einer prognostizierten Verteilungsbilanz von minus 1,6\% wird die Lohnzurückhaltung dabei in Deutschland wieder besonders ausgeprägt sein.

\section{Mindestlöhne}

In allen europäischen Ländern werden Mindestlöhne entweder durch Gesetz und/ oder Tarifverträge festgelegt (Schulten et al. 2006). Innerhalb der EU verfügen 20 von 27 Staaten über einen branchenübergreifenden gesetzlichen Mindestlohn, der eine allgemeine Lohnuntergrenze definiert, die nicht unterschritten werden darf. Während einige EU-Staaten - wie z. B. Frankreich oder die Benelux-Länder - bereits seit mehreren Jahrzehnten praktische Erfahrungen mit gesetzlichen Mindestlöhnen besitzen, haben Irland und Großbritannien erst Ende der 1990er Jahre nationale gesetzliche Mindestlöhne eingeführt. In den meisten Ländern aus Mittel- und Osteuropa (MOE) wurden im Rahmen der Transformationsperiode Anfang der 1990er Jah-

\begin{tabular}{|c|c|c|c|c|c|}
\hline & \multicolumn{2}{|c|}{ Euro'2) } & \multicolumn{2}{|c|}{ KKS3) } & \multirow[t]{2}{*}{ Letzte Erhöhung } \\
\hline & pro Stunde & pro Monat & pro Stunde & pro Monat & \\
\hline Luxemburg & 9,30 & 1.609 & 9,01 & 1.559 & 1. März 2008 \\
\hline Frankreich & 8,71 & 1.324 & 8,11 & 1.233 & 1. Juli 2008 \\
\hline Irland & 8,65 & 1.462 & 6,93 & 1.171 & 1. Juli 2007 \\
\hline Belgien & 8,41 & 1.388 & 7,92 & 1.307 & 1. Mai 2008 \\
\hline Niederlande & 8,33 & 1.357 & 8,02 & 1.307 & 1. Juli 2008 \\
\hline Großbritannien & 6,91 & 1.195 & 6,28 & 1.086 & 1. Oktober 2007 \\
\hline Griechenland & 3,80 & 658 & 4,26 & 738 & 1. Mai 2007 \\
\hline Spanien & 3,59 & 600 & 3,85 & 643 & 1. Januar 2008 \\
\hline Malta & 3,55 & 615 & 4,84 & 838 & 1. Januar 2008 \\
\hline Slowenien & 3,28 & 567 & 4,35 & 752 & 1. März 2008 \\
\hline Portugal & 2,55 & 426 & 2,98 & 497 & 1. Januar 2008 \\
\hline Tschechien & 1,97 & 325 & 3,22 & 530 & 1. Januar 2007 \\
\hline Polen & 1,92 & 332 & 3,20 & 554 & 1. Januar 2008 \\
\hline Estland & 1,61 & 278 & 2,42 & 418 & 1. Januar 2008 \\
\hline Ungarn & 1,61 & 279 & 2,76 & 478 & 1. Januar 2008 \\
\hline Slowakei & 1,54 & 267 & 2,71 & 469 & 1. Oktober 2007 \\
\hline Litauen & 1,34 & 232 & 2,36 & 409 & 1. Januar 2008 \\
\hline Lettland & 1,34 & 231 & 2,21 & 381 & 1. Januar 2008 \\
\hline Rumänien & 0,79 & 136 & 1,39 & 240 & 1. Januar 2008 \\
\hline Bulgarien & 0,65 & 112 & 1,45 & 250 & 1. Januar 2008 \\
\hline \multicolumn{6}{|c|}{$\begin{array}{l}\text { 1) Berechnungen auf der Basis folgender Wochenarbeitszeiten: } 40 \text { Stunden: Estland, Griechenland, Großbritannien, Lettland, } \\
\text { Litauen, Luxemburg, Malta, Poland, Slowenien, Ungarn; } 39 \text { Stunden: Irland, Slowakei; } 38,5 \text { Stunden: Spain, Portugal; } 38 \text { Stunden: } \\
\text { Belgien, Tschechien; } 37,5 \text { Stunden: Niederlande; } 35 \text { Stunden: Frankreich. } \\
\text { 2) Berechnungen von nationalen Währungen in Euro auf der Basis des Wechselkurses vom 10. Juni } 2008 . \\
\text { 3) Berechnungen auf der Basis von Kaufkraftparitäten für } 2006 \text {. }\end{array}$} \\
\hline Quelle: WSI Mindes & & & & & MITTEILUNGEN \\
\hline
\end{tabular}

re neue gesetzliche Mindestlohnregelungen geschaffen, die seither vor dem Hintergrund eher schwach entwickelter Tarifvertragssysteme für die gesamte Lohnentwicklung eine besonders wichtige Rolle spielen.

In den meisten europäischen Ländern erfolgt die Erhöhung der Mindestlöhne unter Beteiligung von Gewerkschaften und Arbeitgeberverbänden nach einem jährlichen oder sogar - wie im Falle der Niederlande - halbjährlichen Turnus und wird an einem bestimmten, immer wiederkehrenden Stichtag vollzogen. In einigen Ländern, wie z. B. Frankreich, Belgien, Luxemburg, Malta sowie seit Jüngstem auch Litauen, werden die gesetzlichen Mindestlöhne zudem automatisch an die Preissteigerungsrate angepasst (EZB 2008a, S. 55f.). In zehn EU-Staaten wurden die Mindestlöhne zuletzt zum 1. Januar 2008 angehoben, in sechs weiteren Ländern erfolgte die Erhöhung im ersten Halbjahr 2008.

Bezogen auf die absolute Höhe des nationalen Mindestlohns lassen sich innerhalb der EU drei Ländergruppen identifizieren (Tabelle 4): Zur ersten Gruppe mit relativ hohen Mindestlöhnen zwischen $6,91 €$ und $9,30 €$ pro Stunde bzw. $1.195 €$ und $1.609 €$ pro Monat gehören die Beneluxstaaten sowie Frankreich, Großbritannien und Irland. Der Wert des britischen Mindestlohns ausgedrückt in Euro ist al- lerdings stark durch die erhebliche Abwertung des britischen Pfundes gegenüber dem Euro verzerrt. Nach der letzten Erhöhung des britischen Mindestlohnes auf 5,52 Pfund im Oktober 2007 entsprach dies noch etwas mehr als $8 € .^{5}$

Eine zweite mittlere Gruppe mit Mindestlöhnen zwischen $3,28 €$ und $3,80 €$ pro Stunde bzw. $567 €$ und $658 €$ pro Monat umfasst die südeuropäischen EUStaaten Spanien, Malta und Griechenland sowie Slowenien. Schließlich finden sich in der dritten Gruppe mit relativ niedrigen Mindestlöhnen zwischen 0,65€ und 2,55€ pro Stunde bzw. $112 €$ und $426 €$ pro Monat mit der Ausnahme von Portugal ausschließlich Staaten aus Mittel- und Osteuropa. Die unterschiedlichen Niveaus der nationalen Mindestlöhne spiegeln zu einem bedeutenden Anteil die unterschiedlichen Lebenshaltungskosten in den einzelnen Ländern wider. Gemessen in Kaufkraftstandards (KKP) reduziert sich das Verhältnis zwischen dem niedrigsten und dem höchsten gesetzlichen Mindestlohn in der EU von 1:14 (gemessen in Euro) auf etwa 1:6.

\footnotetext{
5 Eine weitere Erhöhung des britischen Mindestlohns auf 5,73 Pfund (etwa 7,20€) wurde bereits für den 1. Oktober 2008 beschlossen.
} 


\begin{tabular}{|c|c|c|c|c|c|}
\hline Alte EU-Staaten & Tarifvertraglich ${ }^{1)}$ & Effektiv2) & Neue EU-Staaten & Tarifvertraglich1) & Effektiv2) \\
\hline Belgien & 37,6 & 39,2 & Bulgarien & 40,0 & 41,5 \\
\hline Dänemark & 37,0 & 39,4 & Estland & 40,0 & 40,9 \\
\hline Deutschland & 37,6 & 40,3 & Lettland & 40,0 & 41,7 \\
\hline Finnland & 37,5 & 39,2 & Litauen & 40,0 & 39,8 \\
\hline Frankreich & 35,0 & 39,2 & Malta & 40,0 & 40,4 \\
\hline Griechenland & 40,0 & 40,6 & Polen & 40,0 & 41,3 \\
\hline Großbritannien & 37,3 & 42,5 & Rumänien & 40,0 & 41,3 \\
\hline Irland & 39,0 & 38,9 & Slowakei & 38,8 & 40,9 \\
\hline Italien & 38,0 & 39,2 & Slowenien & 40,0 & 41,4 \\
\hline Luxemburg & 39,0 & 39,9 & Tschechien & 38,0 & 41,4 \\
\hline Niederlande & 37,5 & 38,9 & Ungarn & 40,0 & 40,7 \\
\hline Österreich & 38,8 & 42,4 & Zypern & 38,0 & 40,1 \\
\hline Portugal & 38,2 & 40,2 & & & \\
\hline Schweden & 37,5 & 39,9 & & & \\
\hline Spanien & 37,9 & 40,8 & & & \\
\hline EU 27 & 38,6 & 40,5 & & & \\
\hline \multicolumn{6}{|c|}{$\begin{array}{l}\text { 1) Durchschnittliche tarifvertraglich vereinbarte Wochenarbeitszeit. } \\
\text { 2) Durchschnittliche effektive Arbeitszeit von Vollzeitbeschäftigten. }\end{array}$} \\
\hline \multicolumn{6}{|c|}{ Quelle: Carley (2008b); Romans/Preclin (2008). } \\
\hline
\end{tabular}

In sieben EU-Staaten - darunter die skandinavischen Länder Dänemark, Schweden und Finnland sowie Deutschland, Österreich, Italien und Zypern - existiert bislang kein nationaler gesetzlicher Mindestlohn, sodass die Mindestlohnsicherung hier im Wesentlichen durch Tarifverträge erfolgt. Allerdings verfügen diese Länder zumeist über funktionale Äquivalente, die ihnen eine hohe Tarifbindung sichern und damit ein weitgehend funktionierendes System tarifvertraglicher Mindestlohnsicherung möglich machen: In den skandinavischen Ländern sichert das sogenannte "Gent-System“, in dem die Gewerkschaften die Arbeitslosenversicherung verwalten, einen nach wie vor außerordentlich hohen gewerkschaftlichen Organisationsgrad und damit verbunden eine hohe Tarifbindung. In Österreich führt die Tatsache, dass in den allermeisten Branchen die Unternehmer Pflichtmitglieder in der Wirtschaftskammer sind, zu einer außerordentlich hohen Tarifbindung. Darüber hinaus haben 2007 die Spitzenverbände der österreichischen Arbeitgeber und Gewerkschaften eine Vereinbarung unterzeichnet, in der sie die Tarifvertragsparteien auf sektoraler Ebene auffordern, die tariflichen Mindestlöhne auf mindestens $1.000 €$ pro Monat anzuheben (Hermann/Schulten 2007). Schließlich besteht in Italien ein durch die Verfassung (Artikel 36) abgesichertes „Recht auf einen angemessenen Lohn“" Nach herrschender Rechtsprechung ist dies der Tariflohn. Lediglich in Deutschland existieren derzeit keine funktionalen Äquivalente, die eine flächendeckende Min-

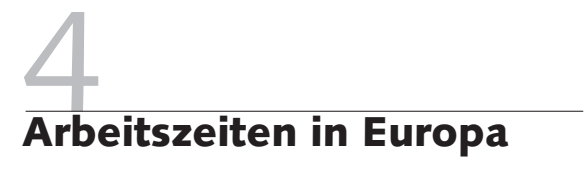

Im Jahr 2007 lag die tarifvertraglich vereinbarte Wochenarbeitszeit innerhalb der EU bei durchschnittlich 38,6 Stunden (Tabelle 5). Da die Dauer der Arbeitszeit in kaum einem europäischen Land auf der tarifpolitischen Agenda stand, sind die tarifvertraglichen Arbeitszeiten gegenüber dem Vorjahr weitgehend konstant geblieben (Carley 2008b). In zehn EU-Staaten, zu denen neben Griechenland und Malta vor allem Länder aus Mittel- und Osteuropa gehören, betrug die vereinbarte Standardwochenarbeitszeit 40 Stunden. In acht Ländern lag die Wochenarbeitszeit zwischen 38 und 39 Stunden und in neun Ländern unterhalb von 38 Stunden. Deutschland bewegte sich mit 37,6 Wochenarbeitsstunden im unteren Mittelfeld. Die kürzeste tarifvertragliche Wochenarbeitszeit bestand der 1990er Jahre die 35-Stunden-Woche als gesetzlich festgeschriebene Regelarbeitszeit gilt. Im Juli 2008 hat die konservative französische Regierung allerdings eine weitgehende Reform der Arbeitszeitgesetzgebung beschlossen, die es den Betrieben wesentlich erleichtert, Überstunden zu vereinbahingegen in Frankreich, wo seit dem Ende ren und damit de facto die 35-Stunden Woche zu unterlaufen (Le Monde, 24. Juli 2008).

In fast allen europäischen Ländern liegen die durchschnittlichen effektiven Wochenarbeitszeiten von Vollzeitbeschäftigten zum Teil deutlich über den tarifvertraglichen Normen (Tabelle 5). Im Jahr 2007 arbeitete ein Arbeitnehmer in der EU durchschnittlich 40,5 Stunden pro Woche und damit 1,9 Stunden mehr als im Tarifvertrag vereinbart wurde. Die längsten effektiven Arbeitszeiten fanden sich mit 42,5 bzw. 42,4 Stunden in Großbritannien und Österreich. In 17 von 27 EU-Staaten lag die effektive Wochenarbeitszeit bei 40 und mehr Stunden. Deutschland befand sich mit durchschnittlich 40,3 Stunden pro Woche genau im EU-Durchschnitt. Die kürzesten effektiven Arbeitszeiten mit einer Dauer von 38,9 Stunden pro Woche existieren in Irland und den Niederlanden, gefolgt von Belgien, Finnland und Frankreich mit 39,2 Stunden. Gegenüber dem Vorjahr sind auch die effektiven Arbeitszeiten weitgehend konstant geblieben.

\section{5 \\ Ausblick: Droht eine Lohn-Preis-Spirale?}

Die Entwicklung der Löhne in Europa hat in den Jahren 2007 und 2008 nachdrücklich an Dynamik gewonnen. Die europäischen Arbeitnehmer drängen immer mehr darauf, ihren angemessenen Anteil am ökonomischen Aufschwung einzufordern und verfügen angesichts rückläufiger Arbeitslosenzahlen über eine deutlich verbesserte Verhandlungsposition. Hinzu kommt seit 2008 der rasche Anstieg der Verbraucherpreise, der zur Sicherung der Reallöhne und darüber hinausgehender Reallohnzuwächse entsprechend höhere Nominallohnsteigerungen nötig macht.

Vor dem Hintergrund steigender Preise und Löhne wird gegenwärtig von zahlreichen Ökonomen das Schreckgespenst der 1970er Jahre mit einer sich gegenseitig hochschaukelnden Lohn-Preis-Spirale an die Wand gemalt. Insbesondere die Europäische Zentralbank (EZB) (2008b, S. 46) wird nicht müde, vor einem durch steigende Löhne induzierten „,verstärkten binnenwirtschaftlichen Inflationsdruck" zu warnen. Der EZB-Präsident Trichet fordert deshalb die „Sozialpartner“ dringend auf, 
sich nicht an dem „aktuell abnormalen Niveau der Inflation" zu orientieren. De facto verlangt er damit von den Arbeitnehmern, Reallohnverluste hinzunehmen (FAZ 2008).

Das Bild der Lohn-Preis-Spirale ist jedoch in mehrfacher Hinsicht problematisch. Zum einen überträgt es die Verantwortung für steigende Preise ausschließlich der Arbeitnehmerseite, während den Unternehmen selbstverständlich zugestanden wird, dass sie steigende Kosten an die Preise weitergeben. Höhere Löhne müssen jedoch keineswegs automatisch zu höheren Preisen führen, sondern können auch durch eine Reduzierung der in den letzten Jahren besonders stark angestiegenen Gewinne finanziert werden. Nach einhelliger Auffassung hat der aktuelle Anstieg der Preise bislang nichts mit den Löhnen, sondern vielmehr mit der Marktmacht von Unternehmen und Spekulanten im Ener- gie- und Nahrungsmittelsektor zu tun, die durch höhere Preise Extragewinne erzielen. Betrachtet man darüber hinaus den für 2008 prognostizierten Anstieg der Löhne, so liegt dieser in den meisten Ländern deutlich unterhalb des nationalen Verteilungsspielraums. Von einer inflationstreibenden Lohnentwicklung kann derzeit in Europa keine Rede sein (ELNEP 2008, S. 26ff.; Janssen 2008).

Die sich gegenwärtig deutlich abschwächende Konjunkturentwicklung liegt vor allem darin begründet, dass die bisherigen Säulen des Aufschwungs, nämlich die Entwicklung der Investitionen sowie der Boom bei den Exporten, merklich an Bedeutung verloren haben. Gleichzeitig hat sich der private Konsum als mögliche dritte Säule eher verhalten entwickelt (Projektgruppe Gemeinschaftsdiagnose 2008, S. 22ff.) Letzteres liegt vor allem an der extrem schwachen Entwicklung des privaten Konsums in Deutschland, der wiederum direkt mit der extrem restriktiven Lohnentwicklung verbunden ist (Hohlfeld 2008).

Da die EU den allergrößten Teil ihres Sozialproduktes innerhalb des europäischen Binnenmarktes erwirtschaftet, wird ihre ökonomische Entwicklung jedoch entscheidend durch die private Nachfrage geprägt (Stockhammer 2007). Angesichts der sich aktuell eintrübenden Konjunktur kommt der Entwicklung der Löhne deshalb eine wichtige Funktion zu, die ökonomische Lage zu stabilisieren. Hierzu ist es wichtig, dass zukünftige Lohnsteigerungen das Reallohnniveau der Arbeitnehmer sichern und darüber hinaus zumindest die Produktivitätszuwächse ausschöpfen. Dies gilt insbesondere für Deutschland, dem als größter Ökonomie in Europa eine besondere Verantwortung zukommt.

\section{ITERATUR}

Bispinck, R. (Hrsg.) (2007): Wohin treibt das Tarifsystem? Hamburg Bispinck, R./Schulten, T. (2008): Aktuelle Mindestlohndebatte: Branchenlösungen oder gesetzlicher Mindestlohn? in: WSI-Mitteilungen 3, S. 151158

Bispinck, R./WSI-Tarifarchiv (2008): Tarifpolitischer Halbjahresbericht Eine Zwischenbilanz der Lohn- und Gehaltsrunde 2008, in: WSI-Mitteilungen 8, S. 455-462

Carley, M. (2008a): Pay Developments - 2007, ElROnline http://www. eurofound.europa.eu/docs/eiro/tn0804019s/tn0804019s.pdf

Carley, M. (2008b): Working Time Developments - 2007, EIROnline, im Erscheinen

European Labour Network for Economic Policy (ELNEP) (2008): Financial Crisis threatens Employment and Growth, Brüssel, Mai

Europäische Kommission (2005): Integrierte Leitlinien für Wachstum und Beschäftigung (2005-2008), KOM (2005) 141 endg. Brüssel, 12.4.2005 Europäische Kommission (2008a): Statistischer Anhang zu „Europäische Wirtschaft“ , Frühjahr 2008, download: http://ec.europa.eu/economy_ finance/publications/publication12534_de.pdf

Europäische Kommission (2008b): Economic Forecasts, Spring 2008, download: http://ec.europa.eu/economy_finance/publications/ publication12530_en.pdf

Eurostat (2008): Juni 2008: Arbeitslosenquote der Eurozone stabil bei 7,3\%, Pressemitteilung 109, vom 31. Juli

Europäischer Gewerkschaftsbund (EGB) (2007): The Coordination of Collective Bargaining 2008. Resolution des EGB Exekutive-Ausschusses, Brüssel, 5.-6. Dezember, download: http://www.etuc.org/a/4459

Europäische Zentralbank (EZB) (2008a): Lohnindexierungsmechanismen in Ländern des Euro-Währungsgebietes in: EZB-Monatsbericht Mai, S. 55-57
Europäische Zentralbank (EZB) (2008b): EZB-Monatsbericht Juli Frankfurter Allgemeine Zeitung (FAZ) (2008): Interview mit dem EZBPräsidenten Jean-Claude Trichet, 17. Juli

Hermann, C./Schulten, T. (2007): Mindestlohn statt Kombilohn, in: WISO - Wirtschafts- und Sozialpolitische Zeitschrift 30, S. 133-151 Hohlfeld, P. (2008): Ende des Aufschwungs Prognose-Update: Deutsche Konjunktur zur Jahresmitte 2008, IMK Report 30, Juni

Janssen, R. (2008): Why Euro Area Wage Developments do not warrant an Increase in Interest Rates, ETUC Collective Bargaining Information Bulletin 3, Brüssel, 30. Juni

Logeay, C./Zwiener, R. (2008): Deutliche Realeinkommensverluste für Arbeitnehmer: Die neue Dimension eines Aufschwungs, in: WSI-Mitteilungen 8, S. 415-422

Projektgruppe Gemeinschaftsdiagnose (2008): Folgen der US-Immobilienkrise belasten Konjunktur, Gemeinschaftsdiagnose Frühjahr 2008, IMK-Report 28, April

Romans, F./Preclin, V. (2008): European Union Labour Force SurveyAnnual Results 2007, Eurostat, Data in focus, Series: Population and Social Conditions 27

Schulten, T. (2007): Europäischer Tarifbericht des WSI - 2006/2007, in: WSI-Mitteilungen 9, S. 475-482

Schulten, T./Bispinck, R./Schäfer, C. (Hrsg.) (2006): Mindestlöhne in Europa, Hamburg

Stockhammer, E. (2007): Funktionale Einkommensverteilung und aggregierte Nachfrage im Euro-Raum, in: Wirtschaft und Gesellschaft 2, S. 175-198

Vring, T. von der (2008): Droht eine europäische Lohnsenkungsspirale?, Friedrich Ebert Stiftung (Hrsg.), Veröffentlichungen des Referates, Reihe „Internationale Politikanalyse “, Juli 